\title{
One misdated sequence of rabbit hemorrhagic disease virus prevents accurate estimation of its nucleotide substitution rate
}

\author{
Allison L Hicks and Siobain Duffy
}

\begin{abstract}
Background: The literature is ripe with phylogenetic estimates of nucleotide substitution rates, especially of measurably evolving species such as RNA viruses. However, it is not known how robust these rate estimates are to inaccuracies in the data, particularly in sampling dates that are used for molecular clock calibration. Here we report on the rate of evolution of the emerging pathogen Rabbit hemorrhagic disease virus (RHDV), which has significantly different rates of evolution for the same outer capsid (VP60) gene published in the literature. In an attempt to reconcile the conflicting data and further elucidate details of RHDV's evolutionary history, we undertook fresh Bayesian analyses and employed jackknife control methods to produce robust substitution rate and time to most recent common ancestor (TMRCA) estimates for RHDV based on the VP60 and RNA-dependent RNA polymerase genes.

Results: Through these control methods, we were able to identify a single misdated taxon, a passaged lab strain used for vaccine production, which was responsible for depressing the RHDV capsid gene's rate of evolution by $65 \%$. Without this isolate, the polymerase and the capsid protein genes had nearly identical rates of evolution: $1.90 \times 10^{-3}$ nucleotide substitutions/site/year, ns/s/y, (95\% highest probability density (HPD) $1.25 \times 10^{-3}-2.55 \times 10^{-3}$ ) and $1.91 \times 10^{-3} \mathrm{~ns} / \mathrm{s} / \mathrm{y}\left(95 \% \mathrm{HPD} 1.50 \times 10^{-3}-2.34 \times 10^{-3}\right)$, respectively.

Conclusions: After excluding the misdated taxon, both genes support a significantly higher substitution rate as well as a relatively recent emergence of RHDV, and obviate the need for previously hypothesized decades of unobserved diversification of the virus. The control methods show that using even one misdated taxon in a large dataset can significantly skew estimates of evolutionary parameters and suggest that it is better practice to use smaller datasets composed of taxa with unequivocal isolation dates. These jackknife controls would be useful for future tip-calibrated rate analyses that include taxa with ambiguous dates of isolation.
\end{abstract}

Keywords: RHDV, Substitution rate, Tip-calibrated, BEAST, Misdated taxon

\section{Background}

The exponentially accumulating sequence data in GenBank have allowed for the publication of hundreds of nucleotide substitution rate estimates for the rapidly evolving RNA viruses. Within a given viral species, published tip-calibrated Bayesian substitution rate estimates are often highly consistent (e.g. Influenza A virus [1-3] and Rabies virus [4-8]). However, for some viruses, such as rabbit hemorrhagic disease virus (RHDV), there is a

\footnotetext{
*Correspondence: duffy@aesop.rutgers.edu

Department of Ecology, Evolution, and Natural Resources, School of Environmental and Biological Sciences Rutgers, The State University of New Jersey, 14 College Farm Rd, New Brunswick, NJ 08901, USA
}

significant discrepancy among published substitution rates [9-13].

RHDV is a positive-sense, single-stranded RNA virus of the family Caliciviridae (genus Lagovirus) and the causal agent of the highly lethal rabbit hemorrhagic disease (RHD). Since the emergence of RHD in China in 1984 [12,14-16], RHDV has spread worldwide and continues to be a growing concern for rabbit meat and fur industries $[17,18]$, as well as a threat to European ecosystems [19]. Heightened surveillance for RHDV has resulted in the identification of rabbit calicivirus (RCV), a nonpathogenic relative of RHDV, in Australia, the United States, and Europe [20-24]. 
RHDV has mean published substitution rate estimates for the outer capsid (VP60) gene ranging from $5.48 \times 10^{-4}$ nucleotide substitutions per site per year (ns/ $\mathrm{s} / \mathrm{y})$ [9] to $2.65 \times 10^{-3} \mathrm{~ns} / \mathrm{s} / \mathrm{y}$ [13], with non-overlapping 95\% highest posterior density (HPD) intervals for these lowest and highest estimates. Not surprisingly, there is also significant variation among the time to most recent common ancestor (TMRCA) estimates for RHDV, with estimated mean coalescent ranging from 1917 [12] to 1967 [13]. As RHD was first described in 1984 $[12,14-16,25]$, the oldest TMRCA implies that the coalescent of virulent RHDV antedates the emergence of RHD by almost seven decades. In the absence of an intermediate reservoir host, it is uncommon for the emergence of an acute, virulent virus to be so extensively decoupled from the appearance of its associated disease [3,26-31]. As a result, there has been significant debate over the timing, location, and mechanisms of RHDV's emergence [12,14,15,25,32,33].

It is possible that the discrepancy among evolutionary rate estimates for RHDV is partially attributable to variation among datasets (uneven temporal or geographic representation, number of taxa, portion of genome analyzed) and/or subtle methodological variations [34-36]. As this range of evolutionary rates is atypical for a gene of a single viral species, a systematic investigation was undertaken to explain variation among published nucleotide substitution rate and TMRCA estimates for RHDV. A combination of jackknifing controls was used to produce robust rate estimates for the VP60 gene and the first estimated substitution rate for RHDV's RNA-dependent RNA polymerase (RdRp) gene. These controls reveal that using one misdated taxon significantly slows the estimated rates, unnecessarily lengthening RHDV's TMRCA. We demonstrate the fragility of tip-calibrated evolutionary analyses and propose jackknife control BEAST runs as a way to identify potential misdated taxa.

\section{Results}

\section{Complete dataset analyses}

The best-fitting nucleotide substitution model for the complete VP60 datasets, regardless of whether or not $\mathrm{RCV}$ isolates were included, was GTR $+\mathrm{I}+\Gamma$ (general time reversible including corrections for invariant sites and a gamma distribution of rate heterogeneity). For the $\mathrm{RdRp}$, the $\operatorname{TrNef}+\Gamma$ model (equal-frequency Tamura-Nei model including a gamma distribution of rate heterogeneity) was selected for the RHDV dataset, while the $S Y M+I+\Gamma$ model (symmetric six-rate model assuming equal base frequencies, including corrections for invariant sites and a gamma distribution of rate heterogeneity) was selected for the RHDV + RCV dataset. The uncorrelated lognormal clock model was determined to be a significantly better fit than the strict clock model for each of the datasets $\left(\log ^{10}\right.$ Bayes factors $\gg 2$ ). Additionally, the 95\% HPDs of the standard deviation for the uncorrelated lognormal relaxed molecular clock rate estimates excluded zero for all of the demographic models, further rejecting a strict molecular clock for these alignments. The Bayesian skyline demographic model was best-fitting for each of the datasets, though it was not significantly better than the constant or exponentially growing population models. However, there was no significant variation in nucleotide substitution rate or TMRCA estimates among demographic models in any of the full datasets.

There was no significant substitution rate variation between RHDV and RHDV + RCV datasets of either gene (Table 1), and the inclusion of the divergent RCV isolates had no significant effects on age estimates for the virulent RHDV clade or its individual lineages (Table 1, Additional files 1,2). The subsequent analyses were performed only on the RHDV datasets.

The maximum clade credibility (MCC) and maximum likelihood (ML) trees for each complete gene dataset were highly congruent (Figures 1, 2). Further, the trees generated for the two different genes were also congruent, with the exception of one taxon, GenBank accession number EF558585, which switched from lineage B in the VP60 tree to lineage $\mathrm{D}$ in the RdRp tree (Figures 1,2). While it has been previously suggested that this taxon has undergone a crossover event at the junction between the $R \mathrm{dRp}$ and VP60 genes [37], it was not detected as a potential recombinant in these single gene analyses.

Table 1 Complete dataset nucleotide substitution rate analyses

\begin{tabular}{|c|c|c|c|c|c|}
\hline & Gene & $\mathrm{N}_{\text {taxa }}$ & $\begin{array}{l}\text { Substitution } \\
\text { Rate }\left(\times 10^{-3}\right)^{*}\end{array}$ & $\begin{array}{c}\text { RHDV } \\
\text { TMRCA* }\end{array}$ & $\begin{array}{c}\text { Corresponding } \\
\text { Year* }\end{array}$ \\
\hline \multirow[t]{2}{*}{ RHDV } & VP60 & 65 & 0.68 & 77 & 1932 \\
\hline & \multicolumn{2}{|c|}{ (+AY269825) } & $(0.40-0.97)$ & $(49-113)$ & (1896-1960) \\
\hline \multirow[t]{2}{*}{ RHDV } & VP60 & 64 & 1.91 & 41 & 1968 \\
\hline & \multicolumn{2}{|c|}{ (-AY269825) } & $(1.50-2.34)$ & $(31-54)$ & (1955-1978) \\
\hline \multirow[t]{2}{*}{ RHDV } & RdRp & 31 & 1.90 & 62 & 1947 \\
\hline & & & $(1.25-2.55)$ & $(37-89)$ & (1920-1972) \\
\hline \multirow{2}{*}{$\begin{array}{l}\text { RHDV } \\
+ \text { RCV }\end{array}$} & VP60 & 104 & 1.01 & 90 & 1919 \\
\hline & & AY269825) & $(0.63-1.44)$ & $(55-142)$ & $(1867-1954)$ \\
\hline \multirow[t]{2}{*}{$R H D V+R C V$} & VP60 & 103 & 2.24 & 45 & 1964 \\
\hline & & AY269825) & $(1.61-2.95)$ & $(33-60)$ & (1949-1976) \\
\hline \multirow[t]{2}{*}{$\mathrm{RHDV}+\mathrm{RCV}$} & RdRp & 33 & 2.33 & 68 & 1941 \\
\hline & & & $(1.19-3.56)$ & $(43-98)$ & (1911-1966) \\
\hline
\end{tabular}

*Mean substitution rate, TMRCA, and corresponding year estimates are shown with upper and lower $95 \%$ HPD bounds. 


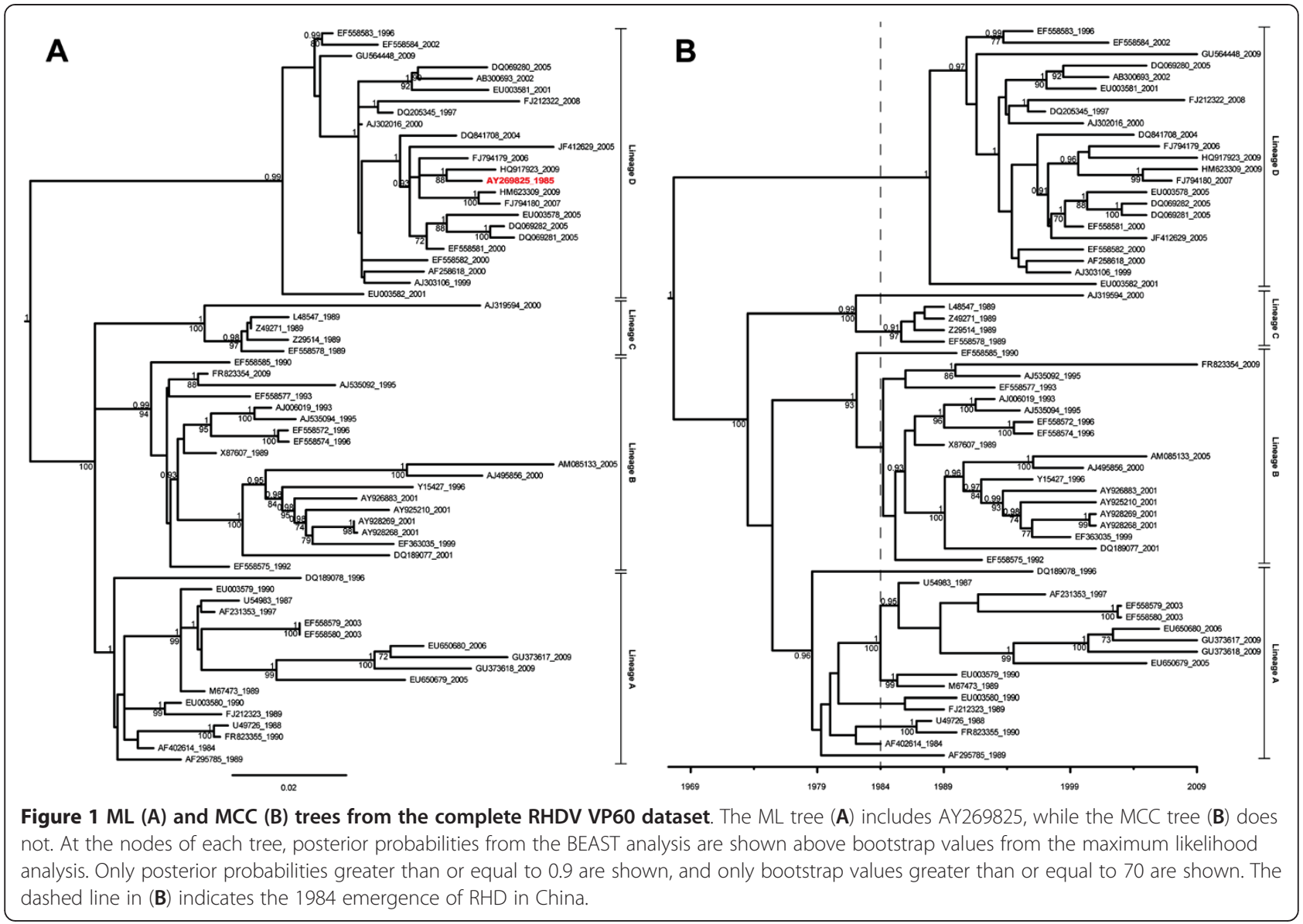

Despite the congruence of tree topology, however, there was a significant difference (non-overlapping 95\% HPDs) between the substitution rate estimates from the complete VP60 and RdRp datasets (Table 1), with the VP60 rate estimate paralleling the lower VP60 rates from the literature $[9,10,12]$, and the RdRp rate paralleling the higher published VP60 rate [13].

To assess the extent of temporal structure in the MCC trees, tip-date randomized controls were run. For both genes, the upper 95\% HPD interval from the tip-date randomized datasets occasionally overlapped the lower 95\% interval of the real dataset substitution rates (Additional file 3). A post-hoc permutation test verified that estimates from the tip-date randomized data sets were different from the substitution rates estimated from the actual dataset ( $\mathrm{p}<0.06$ for VP60, $\mathrm{p}<0.02$ for $\mathrm{RdRp}$ ). Root-to-tip regressions showed moderate correlations (VP60: 0.76, RdRp: 0.70). There is statistical support for temporal structure in each gene's dataset.

\section{Control analyses}

The jackknifed 30 taxa control analysis for the VP60 gene resulted in two distinct groups (Figure 3), roughly corresponding to the two divisions of RHDV rate and TMRCA estimates in literature. By comparing the compositions of the two groups, it was found there was only one taxon (GenBank accession number AY269825) that was present in every dataset from one group and absent from every dataset in the other group. The statistical significance of grouping datasets by the presence or absence of AY269825 is shown in Figure 4. In contrast to the VP60 dataset, the jackknifed 15 taxa control analysis for the RdRp dataset, which did not contain AY269825, yielded one continuous group of substitution rate estimates (Additional file 4).

The n-1 jackknife control analysis for the VP60 gene further implicated AY269825 as having a significant effect on the estimated substitution rate. Only the removal of AY269825 resulted in a substitution rate estimate that was significantly higher than the other $\mathrm{n}-1$ jackknife datasets (Figure 5) and the complete VP60 dataset (Table 1). Removal of AY269825 also resulted in a TMRCA estimate that was substantially more recent than that from the complete dataset (Table 1). For the RdRp, the substitution rates estimated from the $31 \mathrm{n}-1$ jackknife datasets were nearly identical to each other and to the complete dataset (Additional file 5). 


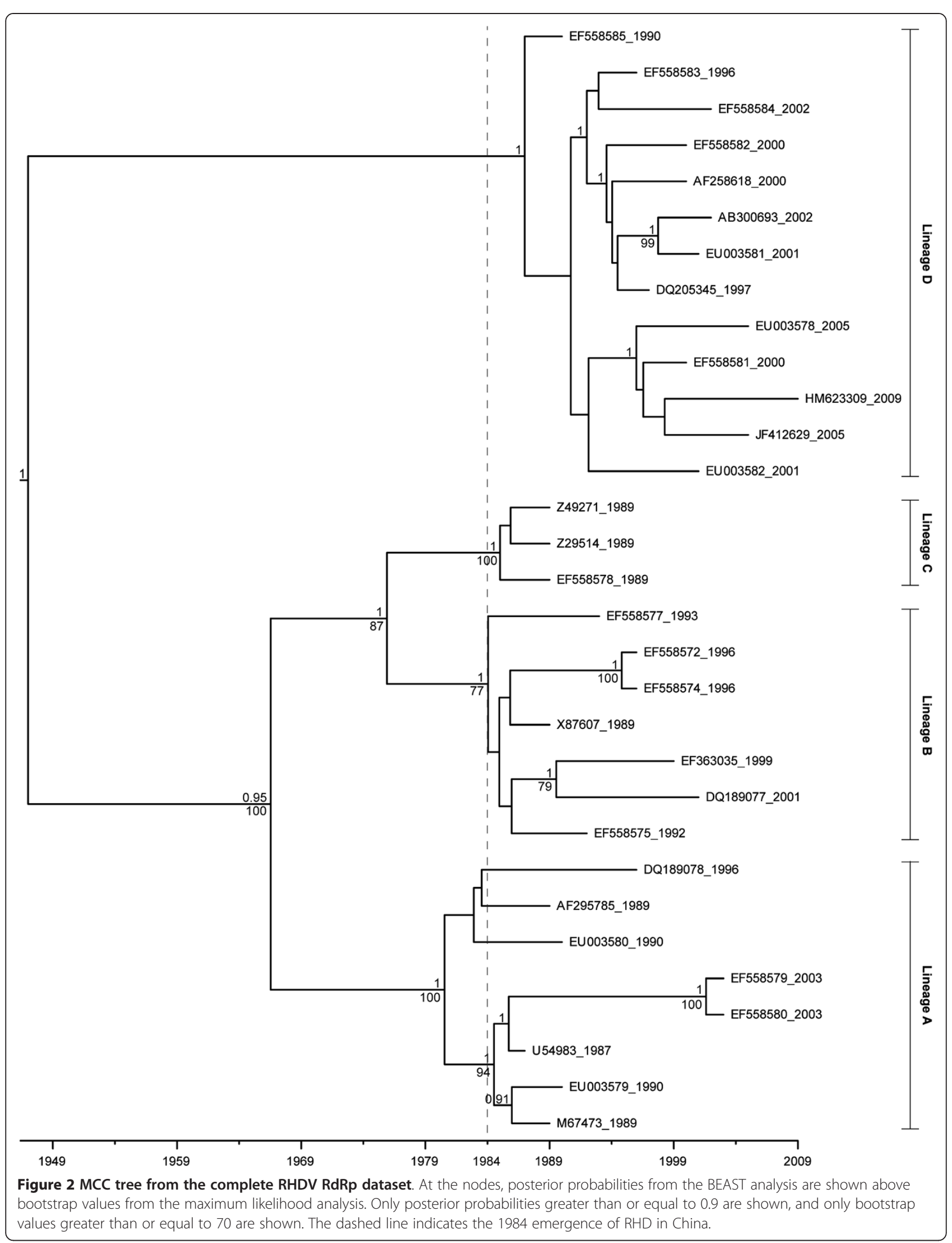


A

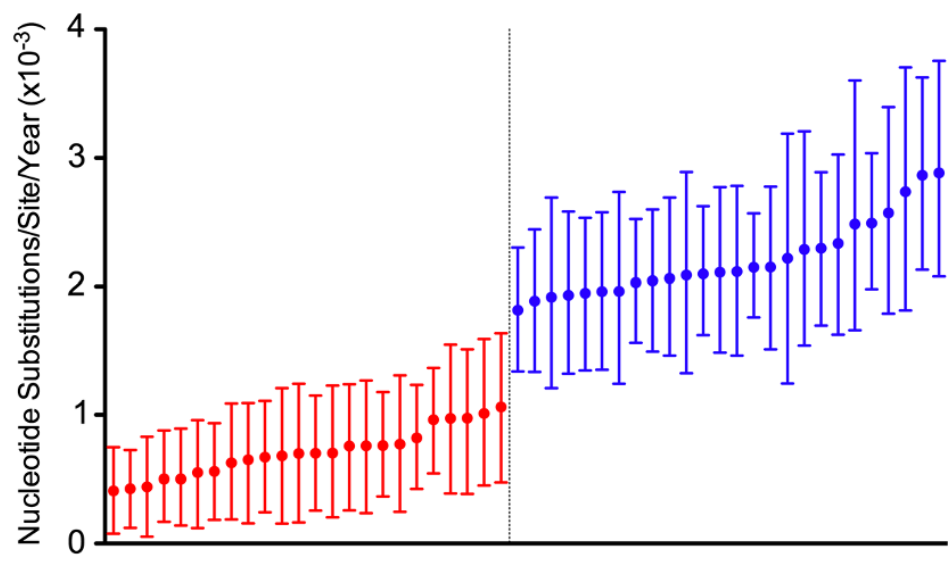

B

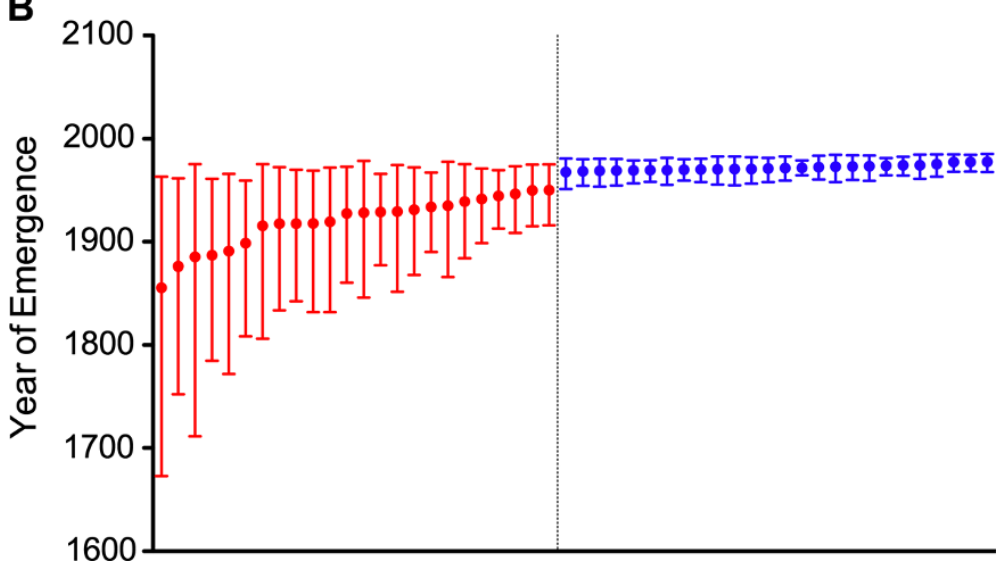

Figure 3 RHDV nucleotide substitution rates (A) and years of emergence (B) estimated from the VP60 jackknife datasets. Mean substitution rates are shown with 95\% HPD intervals for each of the 50 datasets of 30 random taxa. Estimates produced from datasets that included AY269825 are shown in red, while datasets that did not include AY269825 are shown in blue.

AY269825, more formally "NJ/China/1985," has been used in previous analyses with 1985 as the year of isolation $[10,12,15,23,38,39]$. By the leaf-dating method, it was estimated that a more appropriate year would have been 2006 (95\% HPD 2003-2009). A root-to-tip regression of the VP60 ML tree, including AY269825, revealed that both AY269825 and FR823354 were potential outliers (highest residuals, Figure 6).

Running a fresh BEAST analysis for the complete VP60 dataset without AY269825 revealed that there was no overlap between the HPDs of these substitution rate estimates and those of the dataset with AY269826, regardless of the demographic priors used. By comparing the estimated years of emergence of virulent RHDV and its individual lineages from analyses with and without AY269825 (Table 1, Figure 7), it was revealed that while the inclusion of this taxon inflated TMRCA estimates for all lineages, the most conspicuous effect was on the lineage in which it grouped (lineage D). Removal of FR823354, however, had little effect on the estimated
VP60 substitution rate and coalescent (Figure 5; estimated

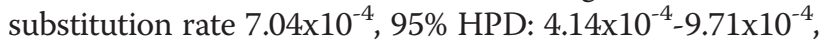
MRCA 1932, 95\% HPD: 1901-1959).

Removal of AY269825 from the complete VP60 dataset did not change the MCC or ML tree topology (Figure 1). However, the tip-date randomization analysis performed on the VP60 dataset without AY269825 revealed stronger evidence of a temporal signal (no overlap of 95\% HPDs from randomized datasets with those of the true dataset; Additional file 3). Similarly, excluding AY269825 from a root-to-tip regression improved the correlation between genetic divergence and time $(r=0.83$, compared to 0.76 when AY269825 was included).

\section{Discussion and conclusions}

The notion that inaccurate specification of dates used for molecular rate calibration could produce misleading results is not a novel one [12,35,40-43]. In the context of RHDV in particular, seven partial VP60 sequences were identified as misdated modern contaminants by 


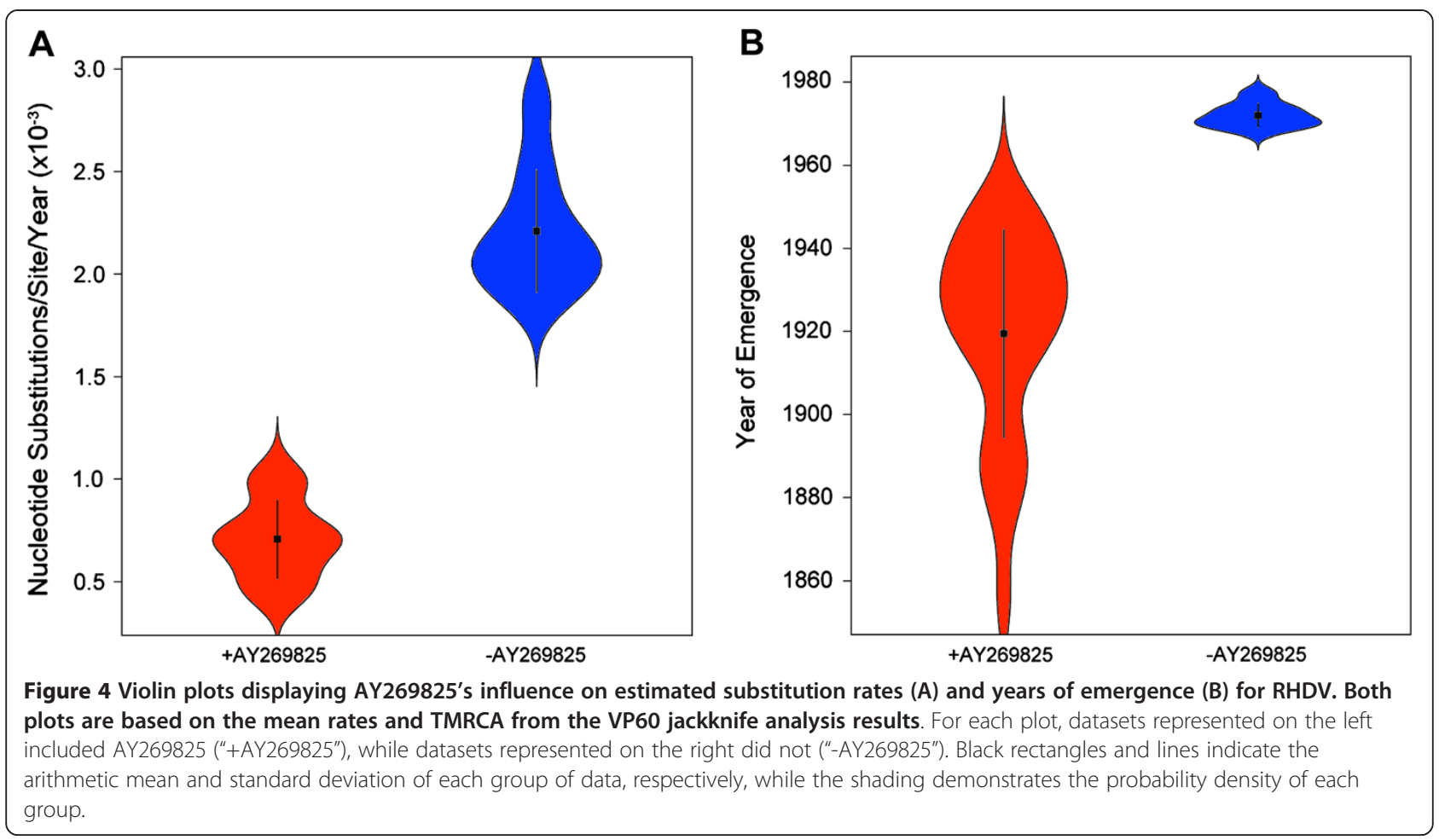

maximum likelihood analysis [12], but were nonetheless included in one BEAST analysis [9], resulting in the slowest substitution rate published for RHDV. It has also been suspected for some time that certain taxa could have a strong influence over inferred phylogenies, and a number of methods have been developed to identify weak clades in phylogenetic trees [44-47] or the highly influential taxa responsible for weakening phylogenetic relationships [48]. However, there is currently no direct method for identifying the presence of a taxon, such as AY269825, that has significant influence over evolutionary rate and TMRCA estimates,

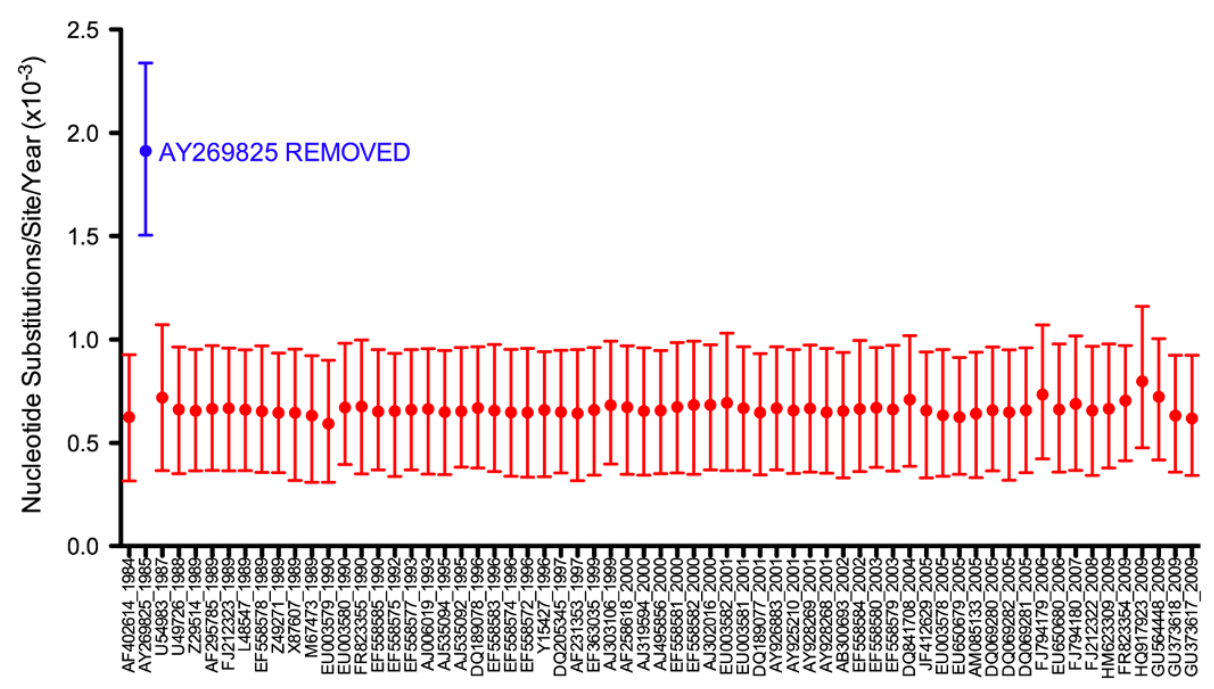

Figure $\mathbf{5}$ RHDV nucleotide substitution rates estimated from the VP60 "n-1" jackknife datasets. Mean substitution rates are shown with 95\% HPD intervals for each of the 65 datasets. Each rate estimate is identified on the x-axis by the GenBank accession number and year of isolation of the taxon removed from its dataset. Estimates produced from datasets that included AY269825 are shown in red, while the dataset that excluded AY269825 is shown in blue. 


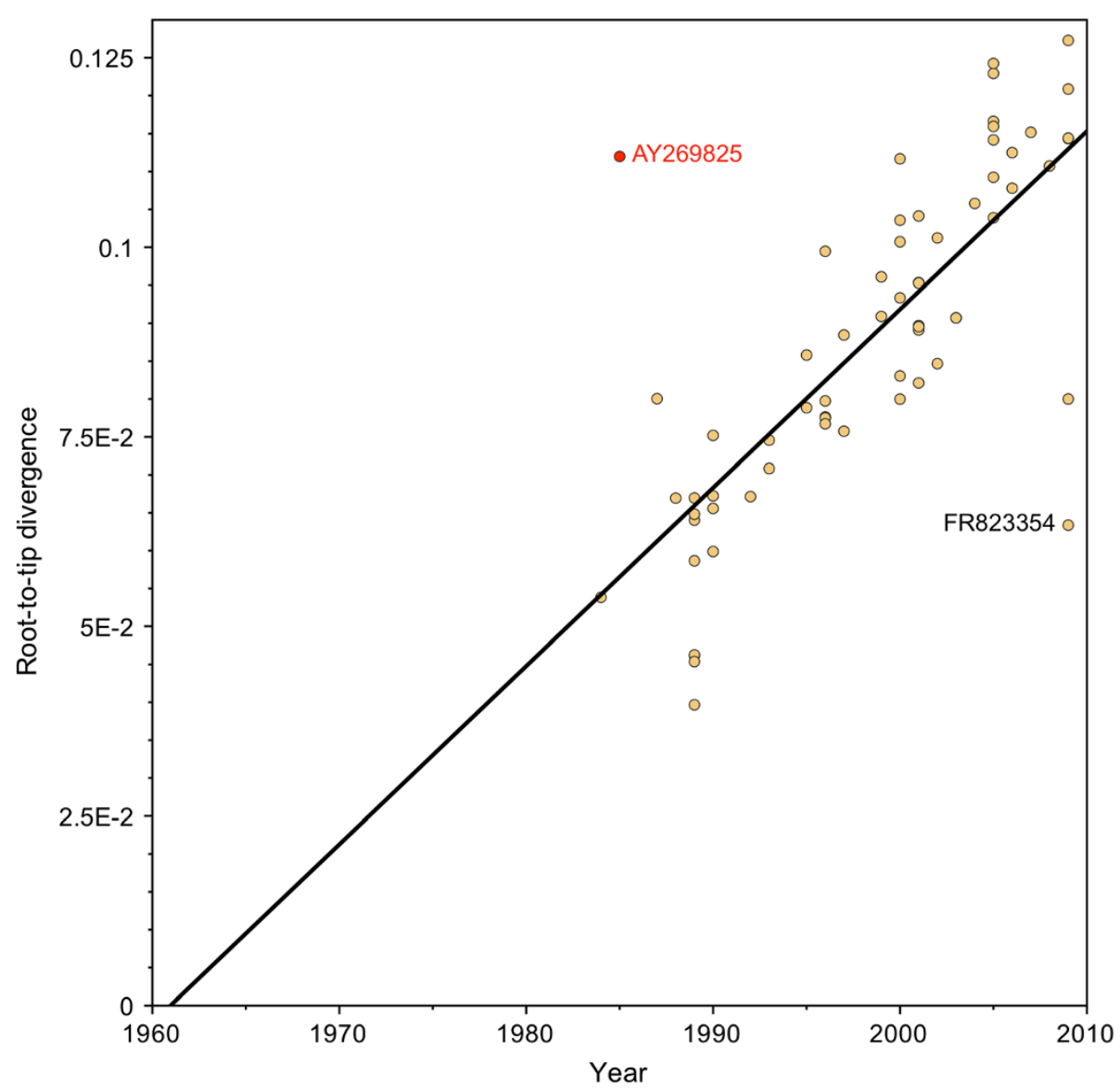

Figure 6 Regression plot for the complete RHDV VP60 dataset. Using the best-fitting root, the regression line indicates the relationship between the root-to-tip genetic distance and the isolation date of each taxon. The two outliers (AY269825 and FR823354, with residual values of 0.055 and 0.049 , respectively) are indicated by GenBank accession number.

while not altering phylogenetic relationships. Further, the influence of misdated taxa on estimates of evolutionary parameters has yet to be extensively examined or quantified.

The comprehensive jackknifing controls used here demonstrate that the Chinese RHDV isolate NJ/China/ 1985, GenBank accession number AY269825, was responsible for dragging down the substitution rate estimate for the VP60 gene by $65 \%$. The Chinese-language paper that described this taxon [49] contained important details about the isolation and handling of this strain. The first paragraph of the methods section (see translation below) revealed that, though it was isolated from nature in 1985, it was maintained in the laboratory for vaccine preparation and was likely not sequenced until much later. The 2003 submission date of AY269825 to GenBank is concordant with the lower bound of the age estimated by the leaf-dating method, as well as its grouping with isolates from 2006-2009 in the MCC and ML trees (Figure 1).
"RHDV NJ85 strain isolate was discovered and characterized byInstitute of Veterinary medicine, Jiangsu Academy of Agricultural Sciences (JAAS), from rabbits raised in an unknown farm in Nanjing City, China, in 1985. Since the discovery, this strain hasbeen maintained in lab rabbits until now. This strain has been usedto prepare potent rabbit hemorrhagic disease vaccine for years Our lab has cloned the gene VP60 in E. coli JM109 and BL21 (DE3)."

The grouping with much more recent isolates could be explained by AY269825's use in RHDV vaccine production. Whether attenuated or improperly inactivated, the strain could have been released into China, and now this lineage can be isolated from other regions of China and Russia ([50,51]; see GenBank file for HM623309 and FJ794179). This is similar to the lab-escape strain that complicated substitution rate estimation for Influenza A virus [40]. Instead of 


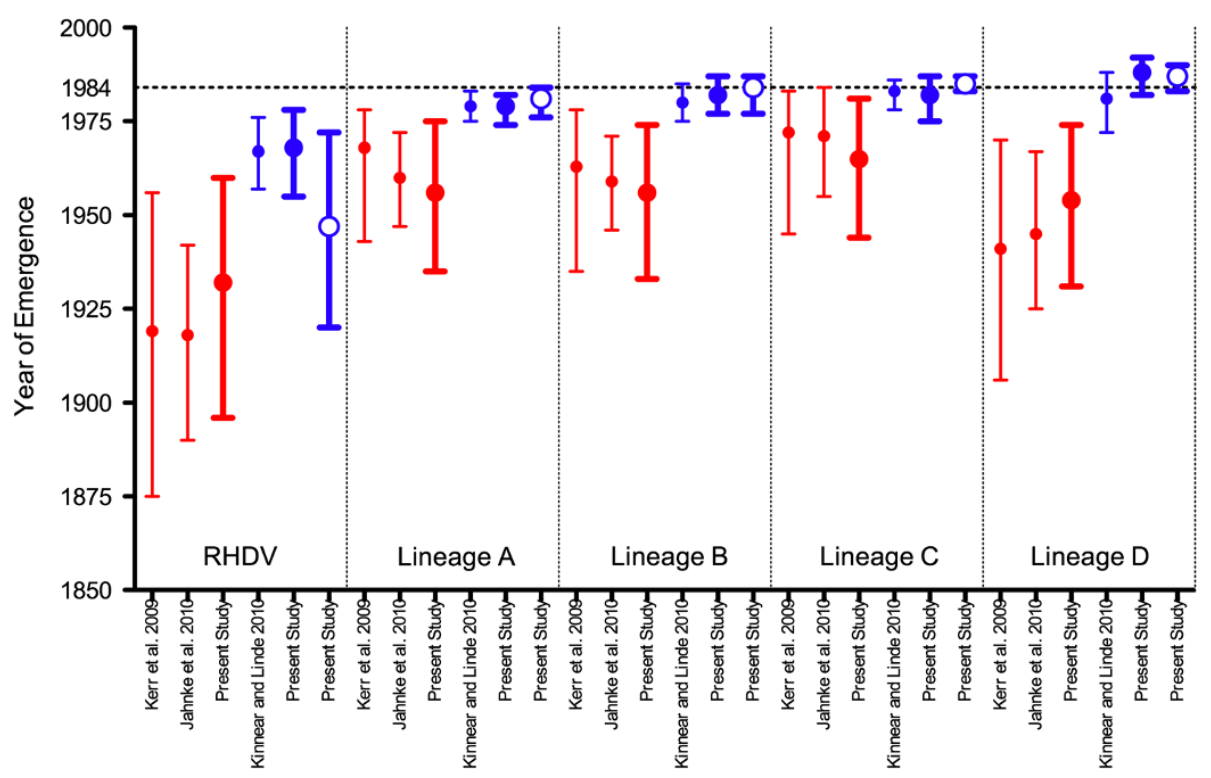

Figure 7 Estimated years of emergence for RHDV lineages. Mean years are shown with 95\% HPD intervals for the entire virulent RHDV clade and each of its four lineages. Closed circles denote estimates based on the VP60 gene, while open circles denote estimates based on the RdRp gene. Years of emergence estimated in the present study are bolded, while those derived from the literature are not. Estimates based on datasets that included AY269825 are shown in red, while those based on datasets that excluded AY269825 are shown in blue. The source of the MCC tree from which each estimate was inferred is indicated on the $x$-axis.

changing many dates of isolation, however, only AY269825 would have to be assigned a different date (removing the four taxa that grouped with AY269825 did not change the estimated substitution rate or TMRCA of RHDV, data not shown). Previous studies have shown that the long-term rate of viral evolution in the lab can mimic the rate in nature [52], so unlike the 20 years of frozen stasis that the Influenza isolate experienced, AY269825 was changing at a rate similar to its wild relatives.

However, consistent results were obtained by excluding this isolate from analyses altogether. Without AY269825, the TMRCA for the entire virulent RHDV complex and each of its lineages is substantially lower, resolving the much of the debate over its puzzling evolutionary history. Indeed, without this misdated taxon, the ancestor of the entire complex is estimated to have existed between 19551978 (Table 1), as few as six years before the 1984 appearance of RHD. These results cannot address whether virulence was a shared trait of the most recent common ancestor of RHDV, or if it evolved independently in multiple lineages. Whenever virulence did evolve, it did not have to go undocumented for several decades $[9,12,14,15,23,25,32,33]$.

While the root-to-tip regression analysis identified AY269825 as potentially deviating from the molecular clock, FR823354, a taxon that did not affect VP60's evolutionary dynamics (Figure 5, [13]), also had a similarly high residual value $(0.055$ of 0.049$)$. This underscores the problem of using congruence with a strict molecular clock as the sole means of assessing the validity of dates of isolation. Deviation from the root-to-tip regression line is expected for viruses with variable rates of evolution, which would be accurately modeled with a relaxed molecular clock BEAST analysis [11,53-57]. Indeed, correlation between genetic distance and time was stronger for the VP60 dataset including the misdated AY269825 taxon than the $R d R p$ dataset, which produced a more trustworthy substitution rate $(\mathrm{r}=0.76$ compared to $r=0.70$ ). The decision to include or exclude taxa based on residuals from a best-fitting line is largely subjective, as there are no guidelines for how common large residuals are in tip-dated viral datasets. In fact, one of the AY269825-containing RHDV datasets had been subjected to this control prior to rate analysis, and the authors did not reject it as an outlier [12]. Finally, rootto-tip regression provides no insight into the magnitude of effect of any taxon on evolutionary estimates. The jackknife controls proposed here focus on detecting taxa that have had a disproportionate effect on the BEAST results, and, in the case of RHDV, offered strong quantitative evidence against including AY269825.

Another interesting finding is that without AY269825, the estimated substitution rate for the VP60 gene is almost identical to that of the RdRp gene, despite the fact that the latter dataset had fewer than half as many taxa (Table 1). Even while the RdRp dataset did not have as strong a temporal signal (Additional file 3 ), probably due 
to the lower number of taxa, it still produced a significantly more accurate substitution rate estimate than datasets with two to three times as many taxa that included just one misdated taxon (Table 1). Further, the estimated substitution rate from our VP60 RHDV + RCV dataset was nearly identical to that from another dataset which contained just 29 taxa (27 RHDV, 1 RCV, did not contain AY269825), including one distantly related European brown hare syndrome virus taxon as an outgroup [13]. This pattern of different sized datasets producing very similar substitution rates is not unique to RHDV. For example, two BEAST analyses of Dengue virus type 2 from datasets of 115 taxa and 67 taxa yielded nearly identical substitution rates [58,59]. Further, in the case of Human parechovirus, three BEAST analyses of three different genomic regions based on datasets with a range of 29-199 taxa also produced nearly identical substitution rates $[55,60]$.

It is evident that assigning years of isolation to taxa should be done with great caution in tip-calibrated rate analyses. These results support favoring data sets with fewer taxa with verifiable dates of isolation over larger data sets with less quality control: additional good data do not swamp out the effects of one badly dated taxon. When researchers are including any ambiguously dated taxa, or when they want to be certain about the effects of each taxon on the rate analysis, jackknife controls provide a clear way to see these effects. As many sequences are added to GenBank without easily accessible papers describing in detail the isolation, passaging and sequencing of each isolate, it is necessary to verify if one or more of the sequences is having a disproportionate influence on the results. We propose $n-1$ jackknifing as one method for researchers using tip-calibrated analyses in BEAST to ensure that a small number of taxa are not spoiling their estimates.

\section{Methods}

Full RHDV VP60 and RdRp gene sequences were downloaded from the GenBank Taxonomy Browser (http:// www.ncbi.nlm.nih.gov/Taxonomy) on 11/16/11. Sequences for each gene with years of isolation available in GenBank or the literature were aligned manually in Se-Al v2.0a11 [61]. Those known to be genetically manipulated or extensively passaged in the lab prior to sequencing were removed from the datasets. Seven United Kingdom isolates that were identified as misdated modern contaminants in a previous ML analysis [12] were excluded because they only covered $30 \%$ of the full VP60 alignment.

As recombination events can lead to over-estimation of nucleotide substitution rates, each dataset was scanned for recombination using seven different algorithms (RDP, GENECONV, Bootscan, MaxChi,
Chimaera, SiScan, and 3seq) implemented in RDP v3.44 [62]. Sequences with recombination signals detected by two or more algorithms were excluded from further analysis (EF558586 was excluded from both gene alignments as a potential recombinant).

GenBank accession numbers and dates of isolation for all taxa used in phylogenetic analyses are given in figures depicting the resulting trees.

\section{Complete dataset analyses}

Modeltest v3.7 [63] was used to determine the best-fit model of nucleotide substitution for each of the alignments (by Akaike's Information Criterion). Estimated nucleotide substitution rates and MCC trees for both the VP60 and RdRp datasets were obtained using BEAST v1.5.4 [64]. Each dataset was run for 200,000,000 generations using two different clock models (strict and uncorrelated lognormal) and three different demographic models (constant, exponential, and Bayesian skyline). The best-fitting clock/demographic model combination was determined using Bayes factors as implemented in Tracer v1.5 [65]. For each set of priors, two independent runs were performed to ensure that the results were replicable. For each dataset, a maximum likelihood analysis was performed using PAUP* v4.0b10 [66] to produce bootstrapsupported (1000 replicates) ML trees for comparison with the MCC trees. MCC trees for the complete VP60 and RdRp datasets, including alignments, are available in TREEBASE (http://purl.org/phylo/treebase/phylows/ study/TB2:S12677).

RHDV has a divergent, avirulent sister group that may be conspecific, RCV. There were 39 (VP60) and two (RdRp) RCV sequences that were initially included in the complete gene datasets. Separate analyses were conducted using RHDV sequences alone.

Previous phylogenies of RHDV have identified four lineages, which are referenced here as lineages A-D, following the nomenclature of Kinnear and Linde (2010). Lineage A is referred to as group 3, lineage B is referred to as group 4, lineage $C$ is referred to as group 2 , and lineage D is referred to as group 1 in Kerr et al. (2009).

To test the strength of the temporal signal in the datasets, BEAST analyses were repeated an additional 10 times for each dataset with sampling years randomized $[41,43,67]$, Additional file 6 . The results of these tip-date randomization runs were then compared to the actual results to ensure that there was significant temporal structure present in the real datasets. Statistical significance was inferred from non-overlapping credibility intervals. In the instance of any overlap of 95\% HPD intervals from the randomized datasets with 95\% HPD interval from the actual dataset, a kind of post-hoc permutation test was employed. For all of the saved states (every 20,000 generations), excluding a $10 \%$ burn-in, the recorded mean 
substitution rate of the actual dataset was compared to those of each of the 10 randomized datasets. The probability that the two posterior distributions were the same was estimated by how often the mean rate from a randomized dataset exceeded the mean rate from the actual dataset. An additional test often used to assess rough temporal structure, root-to-tip regression analysis assuming a strict molecular clock, was conducted for the VP60 dataset using Path-O-Gen v1.3 [68].

\section{Control analyses}

In the first attempt to identify if certain taxa were responsible for the substantial variation in published RHDV rate estimates based on the VP60 gene, the complete VP60 dataset (RHDV-only, 65 taxa) was used to generate 50 smaller datasets, each containing 30 taxa selected using a random number generator (Microsoft Excel, 2008). Each of these jackknifed datasets was run in BEAST, as described above, with the same best-fitting priors from the full dataset. In a parallel analysis, the 31 $R d R p$ taxa were jackknifed into 30 independent subsets of 15 taxa each (exact taxa used for jackknife analyses available in Additional file 7). These smaller datasets were run until all parameters had stable ESS values (>200). Similarity between the mean estimated rates was determined visually by plotting the mean and 95\% HPDs and by using the data to generate violin plots (violinmplot package in $\mathrm{R}[69,70])$.

To further assess the effect of each individual taxon on substitution rate estimates, a jackknife " $n-1$ " analysis was performed in which 65 datasets were generated from the complete VP60 dataset, each with one taxon removed. Each of these n-1 datasets was then run in BEAST, as described above with the same best-fitting priors from the full dataset. Whether a taxon had a statistically significant effect on substitution rate was determined by non-overlapping 95\% HPD intervals with those from the complete VP60 dataset and from the other n-1 jackknife runs. An equivalent n-1 jackknife analysis was also performed on the RdRp dataset.

Any taxon identified as exerting a significant effect on a rate estimate was considered potentially misdated, and the method of leaf-dating via BEAST [71] was employed to determine a more accurate estimate of the suspect taxon's age. Further, any such taxon was subsequently removed from the complete datasets, and the complete dataset analyses described above were repeated for the datasets without the suspect taxon.

\section{Additional files}

Additional file 1: $M C C$ tree produced from the complete RHDV + RCV VP60 dataset (without AY269825). Node bars represent the 95\% HPDs for the node ages. The dashed line indicates the 1984 emergence of RHD in China.
Additional file 2: MCC tree produced from the complete RHDV + RCV RdRp dataset. Node bars represent the 95\% HPDs for the node ages. The dashed line indicates the 1984 emergence of RHD in China.

Additional file 3: RHDV nucleotide substitution rates estimated from the tip-date randomization control analyses. Mean substitution rates are shown with 95\% HPD intervals for the VP60 dataset with AY269825 (65 taxa, left), the VP60 dataset without AY269825 (64 taxa, middle), and the RdRp dataset (31 taxa, right). For each group, the leftmost value is the estimated substitution rate from the actual dataset, while the following 10 values are those from the tip-date randomized datasets.

Additional file 4: RHDV nucleotide substitution rates estimated from the RdRp jackknife datasets. Mean substitution rates are shown with 95\% HPD intervals for each of the 30 datasets of 15 random taxa.

Additional file 5: RHDV nucleotide substitution rates estimated from the RdRp "n-1" jackknife datasets. Mean substitution rates are shown with 95\% HPD intervals for each of the 31 datasets. Each rate estimate is identified on the $x$-axis by the GenBank accession number and year of isolation of the taxon removed from its corresponding dataset.

Additional file 6: RHDV VP60 and RdRp Tip-Date Randomized Datasets. Taxa are listed with the random dates assigned to them for each of the 10 tip-date randomized datasets for the full VP60, with and without AY269825, and the RdRp genes.

Additional file 7: RHDV taxa included in the VP60 and RdRp random jackknife control analyses. Bolded taxa indicate those used in each of the 50 VP60 and 30 RdRp random jackknife datasets.

Competing interests

The authors declare that they have no competing interests.

\section{Acknowledgements}

We thank Eric Ho for providing the Chinese to English translation of the methods excerpt. We thank Edward Holmes and Andrew Kitchen for discussions relating to the RHDV rate discrepancies in the literature. This work was supported by the National Institute of Allergy and Infectious Diseases at the National Institutes of Health (grant number 1R03Al096265-01).

\section{Authors' contributions}

ALH conducted all BEAST and PAUP* analyses and statistical analyses of the results. SD conducted the Path-O-Gen and post-hoc permutation analyses. ALH and SD wrote the paper. All authors read and approved the final manuscript.

Received: 13 March 2012 Accepted: 30 May 2012

Published: 30 May 2012

\section{References}

1. Chen RB, Holmes EC: Avian influenza virus exhibits rapid evolutionary dynamics. Mol Biol Evol 2006, 23:2336-2341.

2. Fourment M, Wood JT, Gibbs AJ, Gibbs MJ: Evolutionary dynamics of the $\mathrm{N} 1$ neuraminidases of the main lineages of influenza $\mathrm{A}$ viruses. $\mathrm{Mol}$ Phylogenet Evol 2010, 56:526-535.

3. Smith GJD, Vijaykrishna D, Bahl J, Lycett SJ, Worobey M, Pybus OG, Ma SK, Cheung $\mathrm{CL}$, Raghwani J, Bhatt $\mathrm{S}$, et al: Origins and evolutionary genomics of the 2009 swine-origin H1N1 influenza A epidemic. Nature 2009, 459:1122-1126.

4. David D, Hughes GJ, Yakobson BA, Davidson I, Un H, Aylan O, Kuzmin IV, Rupprecht CE: Identification of novel canine rabies virus clades in the Middle East and North Africa. J Gen Virol 2007, 88:967-980.

5. Davis PL, Bourhy H, Holmes EC: The evolutionary history and dynamics of bat rabies virus. Infect Genet Evol 2006, 6:464-473.

6. Bourhy H, Reynes JM, Dunham EJ, Dacheux L, Larrous F, Huong VTO, Xu GL, Yan JX, Miranda MEG, Holmes EC: The origin and phylogeography of dog rabies virus. $J$ Gen Virol 2008, 89:2673-2681.

7. Talbi C, Holmes EC, De Benedictis P, Faye O, Nakoune E, Gamatie D, Diarra A, Elmamy BO, Sow A, Adjogoua EV, et al: Evolutionary history and 
dynamics of dog rabies virus in western and central Africa. J Gen Virol 2009, 90:783-791.

8. Ming PG, Yan JX, Rayner S, Meng SL, Xu GL, Tang Q, Wu J, Luo J, Yang XM: A history estimate and evolutionary analysis of rabies virus variants in China. J Gen Virol 2010, 91:759-764

9. Alda F, Gaitero T, Suarez M, Merchan T, Rocha G, Doadrio I: Evolutionary history and molecular epidemiology of rabbit haemorrhagic disease virus in the Iberian Peninsula and Western Europe. BMC Evol Biol 2010, 10:347.

10. Jahnke M, Holmes EC, Kerr PJ, Wright JD, Strive T: Evolution and phylogeography of the nonpathogenic calicivirus RCV-A1 in wild rabbits in Australia. J Virol 2010, 84:12397-12404.

11. Jenkins GM, Rambaut A, Pybus OG, Holmes EC: Rates of molecular evolution in RNA viruses: A quantitative phylogenetic analysis. $J \mathrm{Mol}$ Evol 2002, 54:156-165.

12. Kerr PJ, Kitchen A, Holmes EC: Origin and phylodynamics of rabbit hemorrhagic disease virus. J Virol 2009, 83:12129-12138.

13. Kinnear M, Linde CC: Capsid gene divergence in rabbit hemorrhagic disease virus. J Gen Virol 2010, 91:174-181.

14. Forrester NL, Trout RC, Turner SL, Kelly D, Boag B, Moss S, Gould EA: Unravelling the paradox of rabbit haemorrhagic disease virus emergence, using phylogenetic analysis; possible implications for rabbit conservation strategies. Biol Conserv 2006, 131:296-306.

15. Forrester NL, Abubakr ML, Abu Elzein EME, al-Afaleq AL, Housawi FMT, Moss SR, Turner SL, Gould EA: Phylogenetic analysis of rabbit haemorrhagic disease virus strains from the Arabian Peninsula: Did RHDV emerge simultaneously in Europe and Asia? Virology 2006, 544 (344):277-282.

16. Cooke BD: Rabbit haemorrhagic disease: field epidemiology and the management of wild rabbit populations. Rev Sci Tech Oie 2002, 21:347358

17. McIntosh MT, Behan SC, Mohamed FM, Lu ZQ, Moran KE, Burrage TG, Neilan JG, Ward GB, Botti G, Capucci L, Metwally SA: A pandemic strain of calicivirus threatens rabbit industries in the Americas. Virol J 2007, 4:96.

18. Saunders G, Kay B, Mutze G, Choquenot D: Observations on the impacts of rabbit haemorrhagic disease on agricultural production values in Australia. Wildlife Res 2002, 29:605-613.

19. Delibes-Mateos M, Redpath SM, Angulo E, Ferrerasa P, Villafuerte R: Rabbits as a keystone species in southern Europe. Bio/ Conserv 2007, 137:149-156.

20. Parkes JP, Norbury GL, Heyward RP, Sullivan G: Epidemiology of rabbit haemorrhagic disease (RHD) in the South Island, New Zealand, 1997-2001. Wildlife Res 2002, 29:543-555.

21. Bergin IL, Wise AG, Bolin SR, Mullaney TP, Kiupel M, Maes RK: Novel Calicivirus Identified in Rabbits, Michigan, USA. Emerg Infect Dis 2009, 15:1955-1962

22. Capucci L, Fusi P, Lavazza A, Pacciarini ML, Rossi C: Detection and preliminary characterization of a new rabbit calicivirus related to rabbit hemorrhagic disease virus but nonpathogenic. J Virol 1996, 70:8614-8623.

23. Forrester NL, Trout RC, Gould EA: Benign circulation of rabbit haemorrhagic disease virus on Lambay Island, Eire. Virology 2007, 358:18-22.

24. Strive T, Wright JD, Robinson AJ: Identification and partial characterisation of a new lagovirus in Australian wild rabbits. Virology 2009, 384:97-105.

25. Moss SR, Turner SL, Trout RC, White PJ, Hudson PJ, Desai A, Armesto M, Forrester NL, Gould EA: Molecular epidemiology of rabbit haemorrhagic disease virus. J Gen Virol 2002, 83:2461-2467

26. Walsh PD, Biek R, Real LA: Wave-like spread of Ebola Zaire. Plos Biol 2005, 3:1946-1953.

27. Vijaykrishna D, Smith GJD, Zhang JX, Peiris JSM, Chen H, Guan Y: Evolutionary insights into the ecology of coronaviruses. J Virol 2007, 81:4012-4020.

28. Smith GJD, Bahl J, Vijaykrishna D, Zhang JX, Poon LLM, Chen HL, Webster RG, Peiris JSM, Guan Y: Dating the emergence of pandemic influenza viruses. P Natl Acad Sci USA 2009, 106:11709-11712.

29. Bok K, Abente EJ, Realpe-Quintero M, Mitra T, Sosnovtsev SV, Kapikian AZ, Green KY: Evolutionary Dynamics of Gll.4 Noroviruses over a 34- Year Period. J Virol 2009, 83:11890-11901.

30. Shi M, Lam TTY, Hon CC, Murtaugh MP, Davies PR, Hui RKH, Li J, Wong LTW, Yip CW, Jiang JW, Leung FCC: Phylogeny-Based Evolutionary, Demographical, and Geographical Dissection of North American Type 2 Porcine Reproductive and Respiratory Syndrome Viruses. J Virol 2010, 84:8700-8711.
31. Hanada K, Suzuki $Y$, Nakane $T$, Hirose $O$, Gojobori $T$ : The origin and evolution of porcine reproductive and respiratory syndrome viruses. $\mathrm{Mol}$ Biol Evol 2005, 22:1024-1031

32. Peacock D, Mutze G, Sinclair R, Kovaliski J, Cooke B: Rabbit haemorrhagic disease: Applying Occam's razor to competing hypotheses. Mol Ecol 2012, 21:1038-1041.

33. Gould EA: First case of rabbit haemorrhagic disease in Canada: contaminated flying insect, vs. long-term infection hypothesis. $\mathrm{Mol}$ EcO 2012, 21:1042-1047

34. Heath TA, Hedtke SM, Hillis DM: Taxon sampling and the accuracy of phylogenetic analyses. J Syst Evol 2008, 46:239-257.

35. Ho SYW, Lanfear R, Bromham L, Phillips MJ, Soubrier J, Rodrigo AG, Cooper A: Time-dependent rates of molecular evolution. Mol Ecol 2011, 20:3087-3101

36. Robinson M, Gouy M, Gautier C, Mouchiroud D: Sensitivity of the relative-rate test to taxonomic sampling. Mol Biol Evol 1998, 15:1091-1098.

37. Forrester NL, Moss SR, Turner SL, Schirrmeier H, Gould EA: Recombination in rabbit haemorrhagic disease virus: Possible impact on evolution and epidemiology. Virology 2008, 376:390-396.

38. Esteves PJ, Abrantes J, Carneiro M, Muller A, Thompson G, van der Loo W: Detection of positive selection in the major capsid protein VP60 of the rabbit haemorrhagic disease virus (RHDV). Virus Res 2008, 137:253-256.

39. Oem JK, Lee KN, Roh IS, Lee KK, Kim SH, Kim HR, Park CK, Joo YS: Identification and characterization of rabbit hemorrhagic disease virus genetic variants isolated in Korea. J Vet Med Sci 2009, 71:1519-1523.

40. Wertheim JO: The re-emergence of H1N1 Influenza virus in 1977: A cautionary tale for estimating divergence times using biologically unrealistic sampling dates. PLoS One 2010, 5(6):11184.

41. Ramsden C, Holmes EC, Charleston MA: Hantavirus evolution in relation to its rodent and insectivore hosts: no evidence for codivergence. Mol Biol Evol 2009, 26:143-153.

42. Ho SYW, Phillips MJ, Cooper A, Drummond AJ: Time dependency of molecular rate estimates and systematic overestimation of recent divergence times. Mol Biol Evol 2005, 22:1561-1568.

43. Firth C, Kitchen A, Shapiro B, Suchard MA, Holmes EC, Rambaut A: Using Time-Structured Data to Estimate Evolutionary Rates of Double-Stranded DNA Viruses. Mol Biol Evol 2010, 27:2038-2051.

44. Siddall ME: Another monophyly index: Revisiting the jackknife. Cladistics 1995, 11:33-56

45. Lanyon SM: Detecting Internal Inconsistencies in Distance Data. Syst Zool 1985, 34:397-403.

46. Lapointe FJ, Kirsch JAW, Bleiweiss R: Jackknifing of Weighted Trees Validation of Phylogenies Reconstructed from Distance Matrices. Mol Phylogenet Evol 1994, 3:256-267.

47. Thorley JL, Wilkinson M: Testing the phylogenetic stability of early tetrapods. J Theor Biol 1999, 200:343-344.

48. Mariadassou M, Bar-Hen A, Kishino H: Taxon influence index: assessing taxon-induced incongruities in phylogenetic inference. Syst Biol 2012, 61:337-345.

49. Wang YS, Lu CP, Zhou ZA: High expression of the capsid protein gene of the Chinese early isolate NJ85 of rabbit hemorrhagic disease virus in Escherichia coli. Journal of Agricultural Biotechnology 2004, 12:408-411.

50. Wang F, Hu B, Ren X, Fan Z, Yang L, Xu W, Zhang Z, He K: Expression of the capsid protein of rabbit haemorrhagic disease virus in insect cells and its protective efficacy to rabbits. Chinese Journal of Animal and Veterinary Sciences 2010, 39:1382-1387.

51. Nikolaev AV: New strains of rabbit haemorrhagic disease virus in Russia. Veterinary Medicine Journal 2011, 2:25-28.

52. Harkins GW, Delport W, Duffy S, Wood N, Monjane AL, Owor BE, Donaldson L, Saumtally S, Triton G, Briddon RW, et al: Experimental evidence indicating that mastreviruses probably did not co-diverge with their hosts. Virol J 2009, 6:104

53. Pybus OG, Barnes E, Taggart R, Lemey P, Markov PV, Rasachak B, Syhavong B, Phetsouvanah R, Sheridan I, Humphreys IS, et al: Genetic History of Hepatitis C Virus in East Asia. J Virol 2009, 83:1071-1082

54. Araujo JMG, Nogueira RMR, Schatzmayr HG, Zanotto PMD, Bello G: Phylogeography and evolutionary history of dengue virus type 3. Infect Genet Evol 2009, 9:716-725.

55. Faria NR, de Vries M, van Hemert FJ, Benschop K, van der Hoek L: Rooting human parechovirus evolution in time. BMC Evol Biol 2009, 9:164. 
56. Auguste AJ, Pybus OG, Carrington CVF: Evolution and dispersal of St. Louis encephalitis virus in the Americas. Infect Genet Evol 2009, 9:709-715.

57. Arrigo NC, Adams AP, Weaver SC: Evolutionary Patterns of Eastern Equine Encephalitis Virus in North versus South America Suggest Ecological Differences and Taxonomic Revision. J Virol 2010, 84:1014-1025.

58. Anez G, Morales-Betoulle ME, Rios M: Circulation of Different Lineages of Dengue Virus Type 2 in Central America, Their Evolutionary Time-Scale and Selection Pressure Analysis. PLoS One 2011, 6(11):27459.

59. Sall AA, Faye O, Diallo M, Firth C, Kitchen A, Holmes EC: Yellow Fever Virus Exhibits Slower Evolutionary Dynamics than Dengue Virus. J Virol 2010, 84:765-772.

60. Hicks AL, Duffy S: Genus-Specific Substitution Rate Variability among Picornaviruses. J Virol 2011, 85:7942-7947.

61. Rambaut A: Se-Al: Sequence alignment editor. Version 2.0a11. Edinburgh, UK: Institute of Evolutionary Biology, University of Edinburgh; 2002.

62. Martin DP, Lemey P, Lott M, Moulton V, Posada D, Lefeuvre P: RDP3: a flexible and fast computer program for analyzing recombination. Bioinformatics 2010, 26:2462-2463.

63. Posada D, Crandall KA: MODELTEST: testing the model of DNA substitution. Bioinformatics 1998, 14:817-818.

64. Drummond AJ, Rambaut A: BEAST: Bayesian evolutionary analysis by sampling trees. BMC Evol Biol 2007, 7:214

65. Rambaut A, Drummond AJ: Tracer: MCMC Trace Analysis Tool. Version 1.5. Edinburgh, UK: Institute of Evolutionary Biology, University of Edinburgh; 2007.

66. Swofford DL: PAUP*. Phylogenetic Analysis Using Parsimony (*and Other Methods). Version 4.0b10. Sunderland, MA: Sinauer Associates; 2003.

67. Duffy S, Holmes EC: Validation of high rates of nucleotide substitution in geminiviruses: phylogenetic evidence from East African cassava mosaic viruses. J Gen Virol 2009, 90:1539-1547.

68. Rambaut A: Path-O-Gen: Temporal Signal Investigation Tool. Version 1.3. Edinburgh, UK: Institute of Evolutionary Biology, University of Edinburgh; 2010.

69. R Development Core Team: R: A Language and Environment for Statistical Computing. Version 2.14.1. Vienna, AT: R Foundation for Statistical Computing; 2011.

70. Majeed R: violinmplot: Combination of violin plot with mean and standard deviation. 2010. http://cran.r-project.org/web/packages/violinmplot/ violinmplot.pdf.

71. Shapiro B, Ho SYW, Drummond AJ, Suchard MA, Pybus OG, Rambaut A: A Bayesian Phylogenetic Method to Estimate Unknown Sequence Ages. Mol Biol Evol 2011, 28:879-887.

doi:10.1186/1471-2148-12-74

Cite this article as: Hicks and Duffy: One misdated sequence of rabbit hemorrhagic disease virus prevents accurate estimation of its nucleotide substitution rate. BMC Evolutionary Biology 2012 12:74.

\section{Submit your next manuscript to BioMed Central and take full advantage of:}

- Convenient online submission

- Thorough peer review

- No space constraints or color figure charges

- Immediate publication on acceptance

- Inclusion in PubMed, CAS, Scopus and Google Scholar

- Research which is freely available for redistribution 